moved the right lobe of the thyroid and tied off the left. A cure was apparently effected but within six months the left lobe enlarged and there was a return of all the old symptoms so that the patient was in about the same condition as before the operation. The hydrobromate of quinine was then administered and a very rapid improvement followed and the patient has remained well but occasionally has to take the quinine. In this connection it seems to us that better results would be obtained from operative treatment if a preparatory course of this drug were administered and if it were to be continued after the operation.

We have used thyreoidectin for a considerable time in 12 cases. The powder form of the serum was used in a dose of from 15 gr. to 40 gr. a day, in divided doses. The results were varying, but in no instances have the favorable results recorded by other physicians been obtained. In many instances the symptoms seemed aggravated by the remedy.

We have had no experience with the specific serum treatment, as prepared by Rogers and Beebe, of New York. This may prove more efficacious, but until we more fully understand the action of these agents, it seems to us we should be very cautious in adopting them.

In conclusion we wish to say a few words about the surgical treatment of this disease. We cannot advocate sending early cases to the surgeon. The results of medical treatment are too favorable, from $70 \%$ to $80 \%$ of the cases being cured by it. This is especially true in the rudimentary forms; better in the form which develops gradually than in the form which develops suddenly with full force; better in the old than in the young. But notwithstanding the good results from medical treatment there will always remain a certain number of cases for the surgeon, viz., when the thyroid gland becomes so large that it produces symptoms of compression or when the patient gets rapidly worse on medical treatment. Organic heart changes and bad general condition are surely contra-indications to operation.

\section{STRICTURES OF THE ESOPHAGUS DILATED THROUGH THE ESOPHAGOSCOPE. REPORT OF A CASE.*}

BY J. PAYSON CLARK, M.D., BOSTON.

Since esophagoscopy may be said to be still in its infancy or, at most, early childhood, and only a comparatively few laryngologists have had any experience in its application, it seemed to the writer that the report of the following case might be of interest and value:

Arthur St. P. L., age six, of French Canadian parentage, was brought to the Throat Department of the Massachusetts General Hospital, on June 10, 1907, with the history of having swallowed a small quantity of

* Read before the meeting of the Eastern Section of the American Jaryngological, Rhinological and Otological Society, Boston, Jan. 4, caustic potash about six months before. For a few days after the mishap he could swallow nothing. Then he was able to swallow liquids but not solids until three days before coming to the hospital, during which time he could not swallow liquids. On passing bougies a firm obstruction was encountered about eight inches from the incisor teeth. The patient was then etherized and a Jackson esophagoscope passed. This brought up in an apparently blind pouch or dilatation seven and a half inches from the incisor teeth. The greater distance shown by the bougie was doubtless due to its flexibility. After considerable search no outlet was discorered. After recovery from ether it was found that the patient could swallow liquids with apparent ease.

To facilitate the finding of the lumen of the stricture he was given, on the morning of the thirteenth, silk thread in a glass of water to swallow, the nurse keeping hold of one end of the thread which was then fastened to his cheek with a strip of plaster. He was then etherized and the esophagoscope introduced. The thread could be easily followed as the tube was pushed slowly down until the latter reached the dilatation above the stricture, when the silk thread could be seen disappearing through a minute opening. The stricture was too small to admit bougie No. $15 \mathrm{~F}$. at first, but a No. 13 was passed and then a No. 15. During the next fortnight three or four unsuccessful attempts were made to pass bougies without the aid of the esophagoscope and general anesthesia.

On June 26, the boy was again etherized and guided by the esophagoscope bougie No. 18 F. was easily passed, No. 20 with somewhat more resistance. It was discovered at this time that there was another stricture, of larger caliber than the first had been, about an inch beyond the first. This was also dilated. The use of the silk thread was found unnecessary at this second operation on account of the larger size of the lumen. On June 28, another unsuccessful attempt was made to pass bougies without ether and the tube. On July 1 bougies Nos. 18, 20 and 21 were passed through the esophagoscope under ether anesthesia. The patient could now swallow soft solids. On July 31 bougies up to and including No. $23 \mathrm{~F}$. were passed under the same conditions. There was generally some bleeding when a tight-fitting bougie was used. Again, on Aug. 28, bougies Nos. 20, 21 and 23 were passed in the same manner. The patient was then instructed to report once a month for treatment. Up to the last visit bougies could not be passed through the stricture without the tube. At last accounts the boy was eating without difficulty.

According to the table compiled by Mosher from Starck, the length of the esophagus from the teeth to the cardia in a child of five years of age is $26 \mathrm{~cm}$. For a child of six it would probably be between 26 and $27 \mathrm{~cm}$., or about ten and a half inches. This would bring the first stricture in this case about three inches from the cardia. Dr. Chevalier Jackson ${ }^{1}$ says, "Flexible bougies of $8 \mathrm{~mm}$. in diameter should pass in infants and children up to ten years in age." Bougie No. 23 F. is between 7 and $8 \mathrm{~mm}$. in diameter. It is evident .therefore that the strictures were dilated to approximately the normal caliber of the esophagus for a child of six. An interesting feature of this case was the marked dilatation of the esophagus above the stricture. This large pouch made the

1 Chevalier Jackson: Tracheo-Bronchoscopy, Esophagoscopy and Gastroscopy. St. Louis, Mo., 1907. 
search for the very small lumen of the stricture a very long and tedious one. Jackson says: "Very often this [the lumen of the stricture] will be of almost pin-hole size seen in the flat wall against which the tube mouth is gently pressed. Often prolonged exploration of a diverticulum, or a number of diverticula, will be necessary before the strictural opening is discovered." T".e previous swallowing by the patient of a silk thread, a well-known device connected with older methods of treatment of esophageal strictures, will obviate any prolonged exploration, the white thread forming a beautiful guide to the operator and saving much valuable time.

The reason for the inability in this case to pass flexible bougies even after the strictures had been considerably dilated, seemed to be the relation of the lumen of the stricture to the dilatation of the esophagus, the latter acting like a diverticulum in which the bougie stopped without being able to enter the stricture. This dilatation will doubtless slowly contract now that the condition of the esophagus below has become more normal.

\section{A CASE WITH GRAVE GASTRIC SYMPTOMS OF REFLEX ORIGIN.}

BY RICHARD F. CHASE, M.D., BOSTON,

JOHN T. BOTTOMLEY, M.D., BOSTON.

ON account of the conditions which led to an incorrect diagnosis and the unexpected findings at operation, the following case seems worthy of report: 1907.

Women's Medical Clinic, Boston Dispensary, Jan. 21,

Mrs. M. M., forty-three, does own housework and works out. She has had no illness within the past ten years to confine her to bed. No specific, nor tubercular history could be obtained. No history of trauma to abdomen. Average weight $128 \mathrm{lb}$., present weight $127 \frac{1}{4}$. Color good. Has always had a "weak stomach," but for past sixteen months it has caused more than the usual trouble, while the past six weeks it has been so bad she has been obliged to stop work. The appetite is diminished, thirst normal. Pain in mid-epigastrium, sharp, not radiating, occurs about one hour after eating and lasts about two hours; coarse foods seem to excite it most, soda relieves it. Eructation of gas is excessive and occurs at any time. "Water brash" causes much annoyance. Nausea occurs at time of pain, but she cannot vomit. Hematemesis, none. Bowels constipated for years, and there is excessive intestinal flatulence. Since coarse foods have excited so much pain and discomfort, the patient has restricted her diet to liquids and soft foods. The tongue is clean, teeth fairly good, breath bad at times. She drinks three cups of tea a day, no coffee nor alcoholic liquors.

Physical examination, heart and lungs negative. Both kidneys are prolapsed to $1^{\circ}$. A tumor the size of a filbert is felt at one inch to the right of and below navel; pressure on it excites a sharp pain which is referred to the cardiac end of stomach; tumor is supposed to be at the pylorus. Albumin, 0. Sugar, 0. The symptoms seem to point only to a gastric condition. The patient was given a provisional diet list, antacid tablets, A. S. \& B. pills.

Jan. 24, third day. Weight $125 \frac{1}{4}, 2$ pounds loss. Test breakfast. Amount $=100+$ cc., some free blood. Free $\mathrm{HCl}=27$. Total acidity $=54$. Percussion of the empty stomach, without inflation, showed the greater curvature to be about $1 \frac{1}{2}$ inches below the navel. The stomach was douched.

Jan. 29, eighth day. Weight $123 \frac{1}{4}$, total loss, $4 \mathrm{lb}$. The symptoms are but slightly relieved, the patient feels weaker. Fasting contents for fourteen hours are $100 \mathrm{cc} ., \frac{1}{4}$ food, $\frac{3}{4}$ liquid, some blood; $\mathrm{HCl}=+$. The bowels are well regulated by the A. S. \& B. pills. Stomach douched.

Feb. 1, eleventh day. Weight 1221, total loss, 5 lb. Patient but little relieved and feeling much weaker. The tumor is palpated as at first visit, slightly movable, but not with respiration. The stomach contents, five hours after the ingestion of $1 \frac{1}{2}$ slices toast, 1 soft egg, and 1 cup tea, amounts to 120 cc., $\frac{3}{4}$ food, no visible blood.

The patient was shown to students at this time as a case of probable cancer of the pylorus, and on account of the good physical condition it seemed that a radical operation might be done. She was sent to the Carney Hospital with this diagnosis.

The operation was done by Dr. Bottomley, Feb. 4, 1907. Through a median incision in the epigastric region the stomach, gall bladder, gall ducts and pancreas were examined. With the exception of a very moderate ptosis of the stomach nothing abnormal was found. The stomach was opened, but neither by touch nor by sight could an ulceration be demonstrated The pylorus was normally patent. The kidneys offered nothing abnormal nor did the spleen. Several hard nodules were felt in right iliac region. To obtain better access to these, the incision in the epigastric region was closed and a second incision made in the right iliac region. The nodules were found to be enlarged, calcareous and cheesy glands in the mesentery of the lower ileum. They varied in size, ranging from that of a bean to that of an English walnut. They were removed. The operative recovery was uneventful.

The case is of interest because it illustrates the now well-recognized fact that pyloric spasm, to which, I believe, the epigastric pain experienced by the patient may be attributed, may be caused by irritation of any part of the intestinal tract, embryologically derived from the mid-gut and drawing its chief nerve supply from the sympathetic system.

After the operation the questions arose, Could the findings have caused the gastric symptoms and would the removal of the calcareous glands relieve the symptoms? The fact that there has been no recurrence of symptoms for one year since the operation seems to furnish a sufficiently definite answer in the affirmative.

While the medical diagnosis was erroneous the course pursued with the case, i.e., the advice of operation, proved highly justifiable. During the eleven days' observation, the patient was but little relieved of symptoms and was evidently losing ground rapidly.

The array of symptoms, the history, age, loss of weight, tumor, moderate stasis of gastric contents and blood, was sufficient to lead most any one to a strong suspicion of gastric cancer, and in the vast majority of cases with such symptoms a cancer will be found.

This case was one of the exceptions. 